\title{
Some Remarks on Application of Sandwich Methods in the Minimum Cost Flow Problem
}

\author{
Marta Kostrzewska ${ }^{1}$, Lesław Socha ${ }^{2}$ \\ ${ }^{1}$ Institute of Mathematics, University of Silesia, Katowice, Poland \\ ${ }^{2}$ Faculty of Mathematics and Natural Sciences, Cardinal Stefan Wyszyński University, Warsaw, Poland \\ Email: marta.kostrzewska83@gmail.com, leslawsocha@poczta.onet.pl
}

Received November 27, 2011; revised December 25, 2011; accepted January 12, 2012

\begin{abstract}
In this paper, two new sandwich algorithms for the convex curve approximation are introduced. The proofs of the linear convergence property of the first method and the quadratic convergence property of the second method are given. The methods are applied to approximate the efficient frontier of the stochastic minimum cost flow problem with the moment bicriterion. Two numerical examples including the comparison of the proposed algorithms with two other literature derivative free methods are given.
\end{abstract}

Keywords: Bicriteria Network Cost Flow Problem; Sandwich Algorithms; Efficient Frontier; Stochastic Costs

\section{Introduction}

The network cost flow problems which describe a lot of real-life problems have been studied recently in many Operation Research papers. One of the basic problems in this field are the bicriteria optimization problems. Although there exist exact computation methods for finding the analytic solution sets of bicriteria linear and quadratic cost flow problems (see e.g. [1,2]), Ruhe [3] and Zadeh [4] have shown that the determination of these sets may be very perplexing, because there exists the possibility of the exponential number of extreme nondominated objecttive vectors on the efficient frontier of the considered problems. The fact that efficient frontiers of bicriteria linear and quadratic cost flow problems are the convex curves in $R^{2}$ allows to apply the sandwich methods for a convex curve approximation in this field of optimization (see e.g. [5-8]). However, in some of these algorithms the derivative information is required. A derivative free method was introduced first by Yang and Goh in [8], who applied it to bicriteria quadratic minimum cost flow problems. The efficient frontiers of these problems are approximated by two piecewise linear functions called further approximation bounds, which construction requires solving of a number of one dimensional minimum cost flow problems. Unfortunately, the method introduced by Yang and Goh works under the assumption that the change of the direction of the tangents of the approximated function is less than or equal to $\frac{\pi}{2}$. Also, Siem et al. in [7] proposed an algorithm based only on the function value evaluation, with the interval bisection partition rule and two new iterative strategies for the determination of the new input data point in each iteration. Authors gave the proof of linear convergence of their algorithm.

In this paper we consider the generalized bicriteria minimum cost flow problem. We are interested in minimizing two cost functions, which satisfy some additional assumptions. Two sandwich methods for the approximation of the efficient frontier of this problem are presented. In the first method, based on the algorithm proposed by Siem et al. [7], new points on the efficient frontier are computed according to the chord rule or the maximum error rule by solving proper convex network problems. In the second method, we modify the lower approximation function discussed in [8], what decreases the Hausdorff distance between upper and lower bounds. We give the proofs of the linear convergence property of the first method called the Simple Triangle Algorithm and the quadratic convergence property of the second method called the Trapezium Algorithm.

The paper is organized as follows. In Section 2, we state a nonlinear bicriteria optimization problem that can be treated as a generalized minimum cost flow problem. In Section 3, two new sandwich methods of approximation of the efficient frontier of the stated problem are presented and in Subsection 3.5 the corresponding algorithms are presented. In Section 4, we discuss the convergence of these algorithms. Section 5 includes the information how to use the methodology from Section 2 in the case of the stochastic minimum cost flow problem 
with the moment bicriterion. To illustrate discussed methods in comparison with the algorithms presented in [7] and [8] two numerical examples are given. Finally, Section 6 contains the conclusions and future research direction. Proofs of lemmas and Theorem 2 are given in Appendix.

\section{Problem Statement}

Let $G$ be the directed network with $n$ nodes and $m$ arcs. Let $R_{+}=[0,+\infty)$. We consider the generalized minimum cost flow problem (GMCFP) defined as follows

$$
\begin{aligned}
& \min \left[h_{1}(x), h_{2}(x)\right]^{T} \\
& \text { s.t. } D x=b, \\
& c_{l} \leq x \leq c_{u},
\end{aligned}
$$

where $D \in R^{n \times m}$ is the node-arc incidence matrix, $b \in R^{n}$ is the net outflow of the nodes, vectors $c_{l}, c_{u} \in R_{+}^{m}$ are the lower and upper capacity bounds for the flow $x \in R_{+}^{m}$ and $h_{1}, h_{2}: R^{m} \rightarrow R_{+}$are the cost functions such that $h_{2}(\cdot)$ is a continuous and convex function and $h_{1}(x)=h\left(c^{T} x\right)$, where $c \in R_{+}^{m}$ and $h: R_{+} \rightarrow R_{+}$is a continuous, concave and strictly increasing function. Let

$$
X=\left\{x \in R_{+}^{m}: D x=b, c_{l} \leq x \leq c_{u}\right\}
$$

be the feasible set of problem (1).

According to the concept of Pareto optimality we consider the relations $\leq$ and $<$ in $R^{2}$ defined as follows

$$
\begin{aligned}
& {\left[a_{1}, a_{2}\right]^{T} \leq\left[b_{1}, b_{2}\right]^{T} \Leftrightarrow a_{1} \leq b_{1} \text { and } a_{2} \leq b_{2},} \\
& {\left[a_{1}, a_{2}\right]^{T}<\left[b_{1}, b_{2}\right]^{T} \Leftrightarrow a_{1}<b_{1} \text { and } a_{2}<b_{2} .}
\end{aligned}
$$

Using these definitions in the field of the bicriteria programming, a feasible solution $x \in X$ is called the efficient solution of problem (1) if there does not exist a feasible solution $y \in X$ such that

$$
\left[h_{1}(y), h_{2}(y)\right]^{T} \leq\left[h_{1}(x), h_{2}(x)\right]^{T} .
$$

The set of all efficient solutions and the image of this set under the objective functions are called the efficient set and the efficient frontier, respectively.

Note that the efficient set $X_{E}$ of problem (1) is the same as the efficient set of following problem

$$
\begin{aligned}
& \min \left[h^{-1}\left(h_{1}(x)\right), h^{-1}\left(h_{2}(x)\right)\right]^{T} \\
& \text { s.t. } D x=b, \\
& c_{l} \leq x \leq c_{u} .
\end{aligned}
$$

Moreover, from the fact that $h^{-1}\left(h_{1}(x)\right)=c^{T} x$ for all $x \in X$ and that the function $h^{-1} \circ h_{2}(\cdot)$ is a convex one it follows the next lemma.

\section{Lemma 1} $R^{2}$
Proof: See Appendix.

\section{The New Sandwich Methods}

In this section, we introduce two new sandwich methods for the approximation of the efficient frontier of problem (3).

\subsection{Initial Set of Points}

Let $f_{1}=h^{-1} \circ h_{1}(\cdot)$ and $f_{2}=h^{-1} \circ h_{2}(\cdot)$ and suppose that

$$
P_{k}=\left(f_{1}\left(x^{k}\right), f_{2}\left(x^{k}\right)\right)
$$

for all $k=1,2, \cdots, r$ are $r$ given points on the efficient frontier of problem (1) such that $x^{1}$ and $x^{r}$ are the lexicographical minimum for the first and the second criterion, respectively. Although we need only three given points on the efficient frontier to start the first method called the Simple Triangle Method (STM) and two points to start the second method called the Trapezium Method (TM), the described methodologies work for any number $r$ of initial points, which may be obtained by solving scalarization problems corresponding to problem (3), i.e.

$$
\begin{aligned}
& \min \left(\lambda_{k} f_{1}(x)+\left(1-\lambda_{k}\right) f_{2}(x)\right) \\
& \text { s.t. } x \in X,
\end{aligned}
$$

where $\lambda_{k}=\frac{k-1}{r-1}$ for all $k=1,2, \cdots, r$.

Another possibility is to find lexicographical minima of problem (3) and then to solve $r-2$ convex programing problems with additional equality constraints

$$
c^{T} x=c^{T} x^{1}+\frac{k}{r-1}\left(c^{T} x^{r}-c^{T} x^{1}\right),
$$

where $k=1, \cdots, r-2$. This method gives $r$ points on the efficient frontier with the following property

$$
f_{1}\left(x^{k+1}\right)-f_{1}\left(x^{k}\right)=\frac{1}{r-1}\left(f_{1}\left(x^{r}\right)-f_{1}\left(x^{1}\right)\right)
$$

for all $k=1,2, \cdots, r-1$.

\subsection{Upper Bound}

Suppose that the initial set $P=\left\{P_{1}, \cdots, P_{r}\right\}$ of points on the efficient frontier is given and that the points are ordered according to the first criterion $f_{1}\left(x^{k}\right)<f_{1}\left(x^{k+1}\right)$ for all $k=1, \cdots, r-1$.

The upper approximation function $u^{k}(\cdot)$ of the frontier on the subinterval $\left[f_{1}\left(x^{k}\right), f_{1}\left(x^{k+1}\right)\right]$ called the upper bound is defined as the straight line through the points $P_{k}$ and $P_{k+1}$, that is

$$
u^{k}(a)=f_{2}\left(x^{k}\right)+\frac{f_{2}\left(x^{k+1}\right)-f_{2}\left(x^{k}\right)}{f_{1}\left(x^{k+1}\right)-f_{1}\left(x^{k}\right)}\left(a-f_{1}\left(x^{k}\right)\right)
$$


for $a \in\left[f_{1}\left(x^{k}\right), f_{1}\left(x^{k+1}\right)\right]$.

\subsection{Lower Bounds}

In the algorithms proposed at the end of this section we will use two different definitions of the lower approximation functions called the lower bounds.

\section{Definition 1}

According to [7] the straight lines defined by the points $P_{k-1}$ and $P_{k}$ and the points $P_{k+1}$ and $P_{k+2}$ approximate the frontier from below so the lower bound $l^{k}(\cdot)$ on the interval $\left[f_{1}\left(x^{k}\right), f_{1}\left(x^{k+1}\right)\right]$ may be constructed in the following form

$$
l^{k}(a)= \begin{cases}u^{k-1}(a) & \text { for } a \in\left[f_{1}\left(x^{k}\right), a_{k}\right] \\ u^{k+1}(a) & \text { for } a \in\left[a_{k}, f_{1}\left(x^{k+1}\right)\right],\end{cases}
$$

where $k=2, \cdots, r-2$ and $a_{k}$ is the point of intersecttion of two linear functions $u^{k-1}(\cdot)$ and $u^{k+1}(\cdot)$.

Moreover, we define the lower approximation bound $l^{k}(\cdot)$ on the most left and the most right interval as follows

$$
l^{1}(a)=u^{2}(a) \text { for } a \in\left[f_{1}\left(x^{1}\right), f_{1}\left(x^{2}\right)\right]
$$

and

$$
I^{r}(a)= \begin{cases}u^{r-1}(a) & \text { for } a \in\left[f_{1}\left(x^{r-1}\right), a_{r-1}\right] \\ f_{2}\left(x^{r}\right) & \text { for } a \in\left[a_{r-1}, f_{1}\left(x^{r}\right)\right],\end{cases}
$$

where $a_{r-1}$ is the point of intersection of function $u^{r-1}(\cdot)$ and the constant function $f_{2}\left(x^{r}\right)$.

If we compute new points on the efficient frontier due to the chord rule (see next section), then definition (9) may be modified, see Rote [9], in the following way

$$
I^{k}(a)=\left\{\begin{array}{l}
f_{2}\left(x^{k}\right)+\frac{f_{2}\left(x^{k+1}\right)-f_{2}\left(x^{k-1}\right)}{f_{1}\left(x^{k+1}\right)-f_{1}\left(x^{k-1}\right)}\left(a-f_{1}\left(x^{k}\right)\right) \\
\text { for } a \in\left[f_{1}\left(x^{k}\right), a_{k}\right] \\
f_{2}\left(x^{k+1}\right)+\frac{f_{2}\left(x^{k+2}\right)-f_{2}\left(x^{k}\right)}{f_{1}\left(x^{k+2}\right)-f_{1}\left(x^{k}\right)}\left(a-f_{1}\left(x^{k+1}\right)\right) \\
\text { for } a \in\left[a_{k}, f_{1}\left(x^{k+1}\right)\right],
\end{array}\right.
$$

where $k=2, \cdots, r-2$ and $a_{k}$ is the point of intersection of corresponding two linear functions. Note that the lower bound is constructed by the tangents to $P_{k}$ and $P_{k+1}$.

Moreover, the lower approximation bound $l^{k}(\cdot)$ on the most left and the most right interval are redefined as follows

$$
\begin{gathered}
l^{1}(a)=f_{2}\left(x^{2}\right)+\frac{f_{2}\left(x^{3}\right)-f_{2}\left(x^{1}\right)}{f_{1}\left(x^{3}\right)-f_{1}\left(x^{1}\right)}\left(a-f_{1}\left(x^{2}\right)\right) \\
\text { for } a \in\left[f_{1}\left(x^{1}\right), f_{1}\left(x^{2}\right)\right]
\end{gathered}
$$

and

$$
I^{r}(a)=\left\{\begin{array}{l}
f_{2}\left(x^{r}\right)+\frac{f_{2}\left(x^{r+1}\right)-f_{2}\left(x^{r-1}\right)}{f_{1}\left(x^{r+1}\right)-f_{1}\left(x^{r-1}\right)}\left(a-f_{1}\left(x^{k}\right)\right) \\
\text { for } a \in\left[f_{1}\left(x^{k}\right), a_{k}\right] \\
f_{2}\left(x^{r}\right) \\
\text { for } a \in\left[a_{r-1}, f_{1}\left(x^{r}\right)\right] .
\end{array}\right.
$$

\section{Definition 2}

The simple modification of the definition presented in [8] leads to the following form of lower approximation bound $l_{*}^{k}(\cdot)$ on the interval $\left[f_{1}\left(x^{k}\right), f_{1}\left(x^{k+1}\right)\right]$

$$
I_{*}^{k}(a)=\left\{\begin{array}{l}
u^{k-1}(a) \\
\text { for } a \in\left[f_{1}\left(x^{k}\right), b_{k}\right] \\
f_{2}\left(y^{k}\right)+\frac{f_{2}\left(x^{k+1}\right)-f_{2}\left(x^{k}\right)}{f_{1}\left(x^{k+1}\right)-f_{1}\left(x^{k}\right)}\left(a-f_{1}\left(y^{k}\right)\right) \\
\quad \text { for } a \in\left[b_{k}, c_{k}\right] \\
u^{k+1}(a) \\
\text { for } a \in\left[c_{k}, f_{1}\left(x^{k+1}\right)\right],
\end{array}\right.
$$

where $k=2, \cdots, r-2$, constants $b_{k}$ and $c_{k}$ are the points of intersection of corresponding linear functions and $y^{k}$ is the solution of the following convex network problem (the chord rule problem)

$$
\begin{aligned}
& \min \left(f_{2}(x)-\frac{f_{2}\left(x^{k}\right)-f_{2}\left(x^{k+1}\right)}{f_{1}\left(x^{k}\right)-f_{1}\left(x^{k+1}\right)} f_{1}(x)\right) \\
& \text { s.t. } x \in X .
\end{aligned}
$$

Similar to the previous case, we define the lower approximation bound on the most left and the most right interval as follows

$$
l_{*}^{1}(a)=\left\{\begin{array}{l}
f_{2}\left(y^{1}\right)+\frac{f_{2}\left(x^{2}\right)-f_{2}\left(x^{1}\right)}{f_{1}\left(x^{2}\right)-f_{1}\left(x^{1}\right)}\left(a-f_{1}\left(y^{1}\right)\right) \\
\text { for } a \in\left[f_{1}\left(x^{1}\right), c_{1}\right] \\
u^{2}(a) \\
\text { for } a \in\left[c_{1}, f_{1}\left(x^{2}\right)\right]
\end{array}\right.
$$

and 


$$
l_{*}^{r}(a)= \begin{cases}u^{r-1}(a) & \text { for } a \in\left[f_{1}\left(x^{r-1}\right), b_{r-1}\right] \\ f_{2}\left(x^{r}\right) & \text { for } a \in\left[b_{r-1}, f_{1}\left(x^{r}\right)\right] .\end{cases}
$$

Moreover, these definitions may be modified like in (12) using the tangents in points $P_{2}, \cdots, P_{r-1}$ in the following form

$$
l_{*}^{k}(a)=\left\{\begin{array}{c}
f_{2}\left(x^{k}\right)+\frac{f_{2}\left(x^{k+1}\right)-f_{2}\left(x^{k-1}\right)}{f_{1}\left(x^{k+1}\right)-f_{1}\left(x^{k-1}\right)}\left(a-f_{1}\left(x^{k}\right)\right) \\
\text { for } a \in\left[f_{1}\left(x^{k}\right), b_{k}\right] \\
f_{2}\left(y^{k}\right)+\frac{f_{2}\left(x^{k+1}\right)-f_{2}\left(x^{k}\right)}{f_{1}\left(x^{k+1}\right)-f_{1}\left(x^{k}\right)}\left(a-f_{1}\left(y^{k}\right)\right) \\
\text { for } a \in\left[b_{k}, c_{k}\right] \\
f_{2}\left(x^{k+1}\right)+\frac{f_{2}\left(x^{k+2}\right)-f_{2}\left(x^{k}\right)}{f_{1}\left(x^{k+2}\right)-f_{1}\left(x^{k}\right)}\left(a-f_{1}\left(x^{k+1}\right)\right) \\
\text { for } a \in\left[c_{k}, f_{1}\left(x^{k+1}\right)\right]
\end{array}\right.
$$

and

$$
I_{*}^{1}(a)=\left\{\begin{array}{c}
f_{2}\left(y^{1}\right)+\frac{f_{2}\left(x^{2}\right)-f_{2}\left(x^{1}\right)}{f_{1}\left(x^{2}\right)-f_{1}\left(x^{1}\right)}\left(a-f_{1}\left(y^{1}\right)\right) \\
\text { for } a \in\left[f_{1}\left(x^{1}\right), c_{1}\right] \\
f_{2}\left(x^{2}\right)+\frac{f_{2}\left(x^{3}\right)-f_{2}\left(x^{1}\right)}{f_{1}\left(x^{3}\right)-f_{1}\left(x^{1}\right)}\left(a-f_{1}\left(x^{2}\right)\right) \\
\text { for } a \in\left[c_{1}, f_{1}\left(x^{2}\right)\right]
\end{array}\right.
$$

and

$$
l_{*}^{r}(a)=\left\{\begin{array}{l}
f_{2}\left(x^{r}\right)+\frac{f_{2}\left(x^{r+1}\right)-f_{2}\left(x^{r-1}\right)}{f_{1}\left(x^{r+1}\right)-f_{1}\left(x^{r-1}\right)}\left(a-f_{1}\left(x^{k}\right)\right) \\
\text { for } a \in\left[f_{1}\left(x^{r-1}\right), b_{r-1}\right] \\
f_{2}\left(x^{r}\right) \\
\text { for } a \in\left[b_{r-1}, f_{1}\left(x^{r}\right)\right] .
\end{array}\right.
$$

If the approximation bounds $u^{k}(\cdot)$ and $l^{k}(\cdot)$ or $l_{*}^{k}(\cdot)$ are constructed for each $k=1, \cdots, r-1$, then we define the upper approximation function $u(\cdot)$ of the efficient frontier of problem (3) in the following way

$$
u(a)=u^{k}(a) \text { for } a \in\left[f_{1}\left(x^{k}\right), f_{1}\left(x^{k+1}\right)\right]
$$

and the lower approximation function due to equality

$$
l(a)=l^{k}(a) \text { for } a \in\left[f_{1}\left(x^{k}\right), f_{1}\left(x^{k+1}\right)\right]
$$

or

$$
l_{*}(a)=l_{*}^{k}(a) \text { for } a \in\left[f_{1}\left(x^{k}\right), f_{1}\left(x^{k+1}\right)\right]
$$

for all $k=1, \cdots, r-1$.

We note that after a small modification of the definition of the lower approximation function in the most right interval, any convex function may be approximated by the lower and upper bounds defined in this subsection.

\subsection{Error Analysis}

Suppose that the approximation bounds have been built and let $\delta=\max \left\{\delta_{1}, \cdots, \delta_{r-1}\right\}$, where $\delta_{k}$ denotes the approximation error on the interval $\left[f_{1}\left(x^{k}\right), f_{1}\left(x^{k+1}\right)\right]$. We consider three different error measures called the Maximum error measure (Maximum vertical error measure) $\left(\delta_{k}^{M}\right)$, the Hausdorff distance measure $\left(\delta_{k}^{H}\right)$ and the Uncertainty area measure $\left(\delta_{k}^{U}\right)$ (see [7])

$$
\begin{gathered}
\delta_{k}^{M}=\max _{a \in\left[f_{1}\left(x^{k}\right), f_{1}\left(x^{k+1}\right)\right]}\left|u_{k}(a)-l_{k}(a)\right|, \\
\delta_{k}^{H}=\max \left\{\sup _{v \in L} \inf _{w \in U}\|v-w\|, \inf _{w \in U} \sup _{v \in L}\|v-w\|\right\},
\end{gathered}
$$

where

$$
\begin{gathered}
U=\left\{\left(a, u_{k}(a)\right): a \in\left[f_{1}\left(x^{k}\right), f_{1}\left(x^{k+1}\right)\right]\right\}, \\
L=\left\{\left(a, l_{k}(a)\right): a \in\left[f_{1}\left(x^{k}\right), f_{1}\left(x^{k+1}\right)\right]\right\}
\end{gathered}
$$

and

$$
\delta_{k}^{U}=\int_{f_{1}\left(x^{k}\right)}^{f_{1}\left(x^{k+1}\right)}\left|u_{k}(a)-l_{k}(a)\right| \mathrm{d} a .
$$

If a measure $\delta$ (one of $\delta^{M}, \delta^{H}, \delta^{U}$ ) does not satisfy a desired accuracy, we choose $k \in\{1, \cdots, r\}$ for which $\delta=\delta_{k}$ and we determine the new point $P^{*}=\left(f_{1}\left(x^{*}\right), f_{2}\left(x^{*}\right)\right)$ on the efficient frontier of problem (3) such that $f_{1}\left(x^{*}\right) \in\left[f_{1}\left(x^{k}\right), f_{1}\left(x^{k+1}\right)\right]$. New points on the efficient frontier may be computed according to the chord rule or the maximum error rule, that is by solving the optimization problem (16) or the following problem (the maximum error rule problem)

$$
\begin{aligned}
& \min f_{2}(x) \\
& \text { s.t. } x \in X, \\
& f_{1}(x)=a_{k},
\end{aligned}
$$

where $a_{k}$ is the point of intersection of linear functions $u^{k-1}(\cdot)$ and $u^{k+1}(\cdot)$. Note that if we construct the lower bound due to definition (15), then the chord rule problem (16) has been already solved.

After the determination of the new point, we rebuild 
the set $P$ of given points on efficient frontier due to following equality

$$
P_{i}= \begin{cases}P_{i} & \text { for } i<k \\ P^{*} & \text { for } i=k \\ P_{i-1} & \text { for } i>k,\end{cases}
$$

then we construct new upper and lower bounds and repeat the procedure until we obtain an error $\delta$ smaller than the prescribed accuracy.

The next lemma describes the relation between the approximation bounds of the efficient frontiers of problems (1) and (3).

\section{Lemma 2}

Let $l(\cdot)$ and $u(\cdot)$ be the lower and upper approximation bounds of the efficient frontier of problem (3) built due to the definitions (8) and (9) or (8) and (15), then $\bar{l}(\cdot)=h \circ l \circ h^{-1}(\cdot)$ and $\bar{u}(\cdot)=h \circ u \circ h^{-1}(\cdot)$ are the lower and upper approximation bounds of the efficient frontier of problem (1).

Moreover, the following inequality is satisfied

$$
\bar{u}\left(h_{1}(x)\right)-\bar{l}\left(h_{1}(x)\right) \leq A\left[u\left(f_{1}(x)\right)-l\left(f_{1}(x)\right)\right]
$$

for all the efficient solutions $x \in X_{E}$ and $A=h_{2}\left(x^{1}\right)$.

Proof: See Appendix.

From Lemma 2 follows that in order to obtain the Maximum error between upper and lower approximation bounds $\bar{l}(\cdot)$ and $\bar{u}(\cdot)$ of problem (1) smaller than or equal to the accuracy parameter $\epsilon$, we need to build the approximation bounds $l(\cdot)$ and $u(\cdot)$ of problem (3) for which the Maximum error is smaller than or equal to $\frac{\epsilon}{A}$.

\subsection{Algorithms}

We present two algorithms described in this section.

\subsubsection{The Simple Triangle Algorithm (STA)}

Input: Introduce an accuracy parameter $\epsilon>0$ and an initial set of points on the efficient frontier $P=\left\{P_{1}, P_{2}, P_{3}\right\}$.

Step 1. Calculate lower and upper bounds $l(\cdot), u(\cdot)$ and error $\delta$. Check if $\delta>\epsilon$, then go to Step 2, otherwise stop.

Step 2. Choose interval $\left[f_{1}\left(x^{k}\right), f_{1}\left(x^{k+1}\right)\right]$ for which the maximum error is achieved. Solve the quadratic problem (16) or (28) to obtain the new point $P^{*}$. Update set $P$, lower and upper bounds $l(\cdot), u(\cdot)$ and error $\delta$. Go to Step 3.

Step 3. Check if $\delta>\epsilon$, then go to Step 2, otherwise stop.

\subsubsection{The Trapezium Algorithm (TA)}

Input: Introduce an accuracy parameter $\epsilon>0$ and an initial set of points on the efficient frontier $P=\left\{P_{1}, P_{2}\right\}$.

Step 1. Solve problem (16) and calculate lower and upper bounds $l_{*}(\cdot), u(\cdot)$ and error $\delta$. Check if $\delta>\epsilon$, then go to Step 2, otherwise stop.

Step 2. Choose interval $\left[f_{1}\left(x^{k}\right), f_{1}\left(x^{k+1}\right)\right]$ for which the maximum error is achieved. The new point $P^{*}=\left(f_{1}\left(y^{k}\right), f_{2}\left(y^{k}\right)\right)$. Update set $P$, solve problem (13), calculate lower and upper bounds $l_{*}(\cdot), u(\cdot)$ and error $\delta$. Go to Step 3.

Step 3. Check if $\delta>\epsilon$, then go to Step 2, otherwise stop.

The geometric illustration of STA and TA is given in Figure 1 and Figure 2, respectively. In Figure 1(a), there is an illustration of an efficient frontier and the corresponding lower and upper bounds determined by three initial points

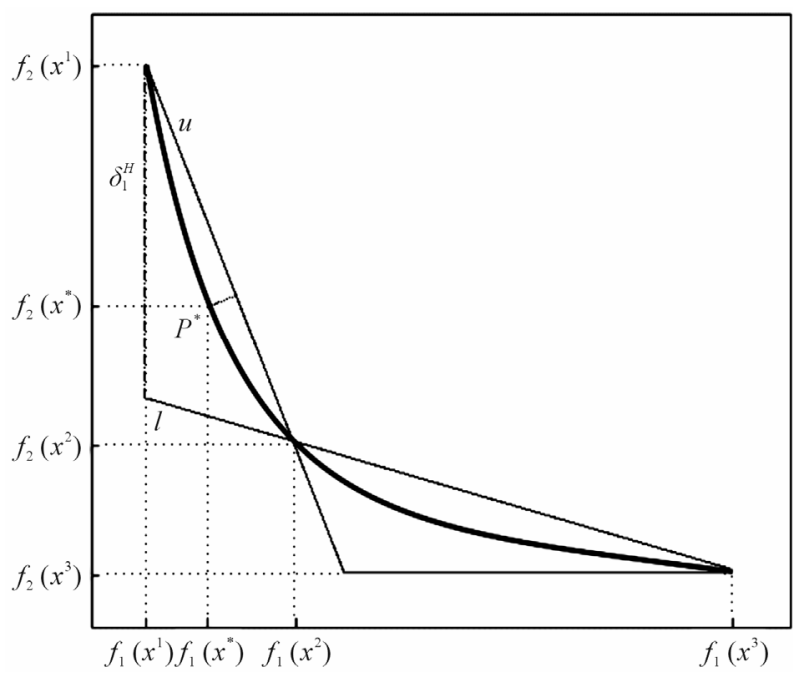

(a)

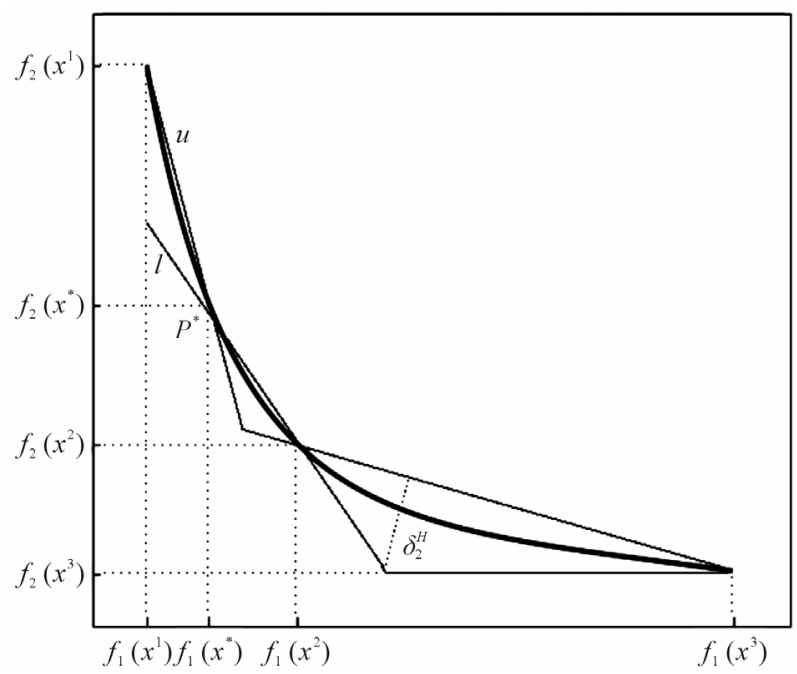

(b)

Figure 1. Lower and upper bounds built due to STA with the chord rule. 


$$
\left(f_{1}\left(x^{1}\right), f_{2}\left(x^{1}\right)\right),\left(f_{1}\left(x^{2}\right), f_{2}\left(x^{2}\right)\right),\left(f_{1}\left(x^{3}\right), f_{2}\left(x^{3}\right)\right) .
$$

Here one can observe that in the interval

$\left[f_{1}\left(x^{1}\right), f_{1}\left(x^{2}\right)\right]$ the Hausdorff measure $\delta_{1}^{H}$ is the greatest. It means that we have to introduce a new point $P^{*}=\left(f_{1}\left(x^{*}\right), f_{2}\left(x^{*}\right)\right)$ into the efficient frontier and determine new corresponding lower and upper bounds, what is illustrated in Figure 1(b). Similar considerations are illustrated in Figures 2(a) and 2(b).

In Figure 3 and Figure 4 we see the illustration of STA and TA which lower bounds built due to definitions (9), (12) and (15), (19), respectively.

In Section 4 we study the convergence of described algorithms.

\section{Convergence of the Algorithms}

In this section we present the convergence results of presented algorithms based on proofs given in Rote [9]

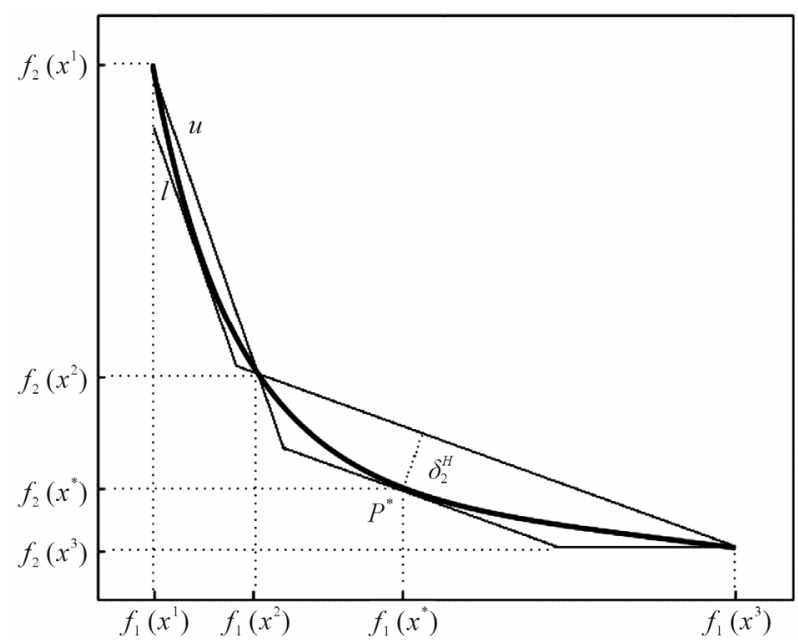

(a)

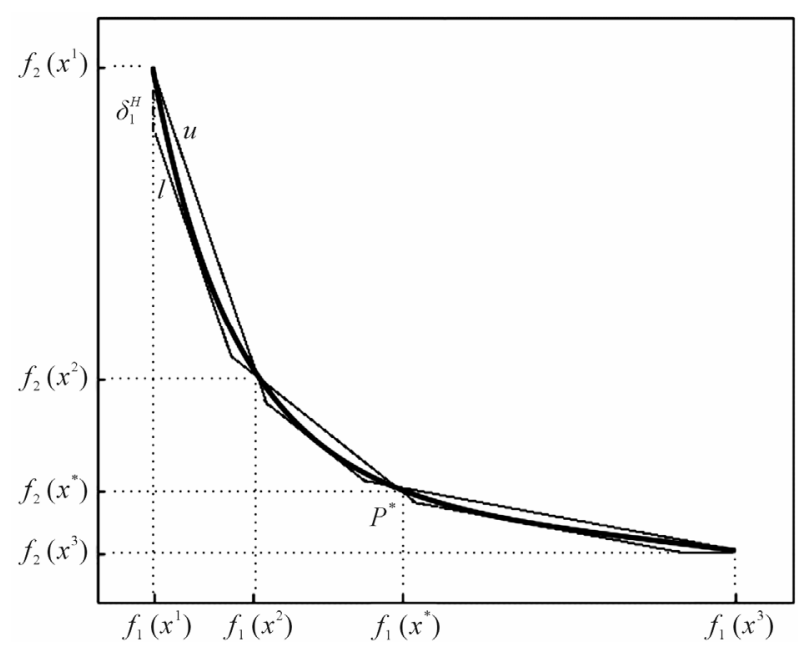

(b)

Figure 2. Lower and upper bounds built due to TA.

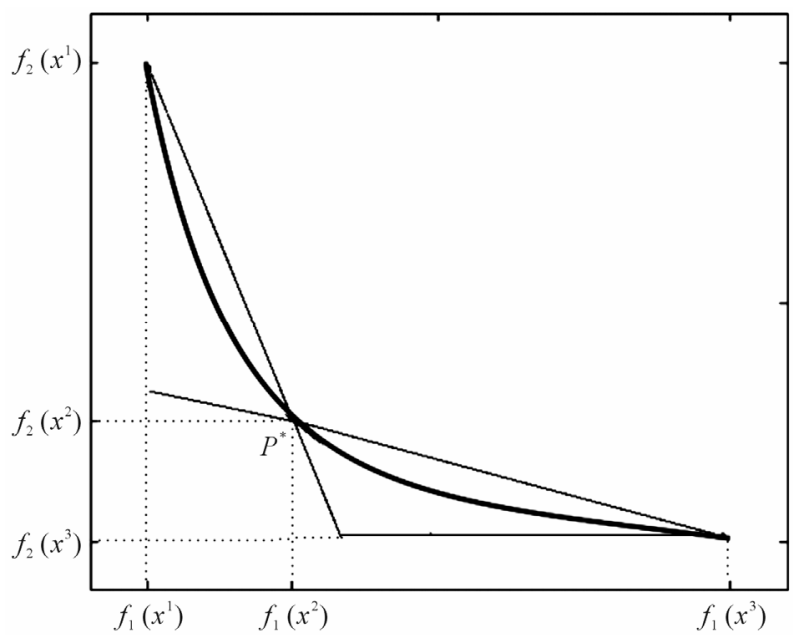

(a)

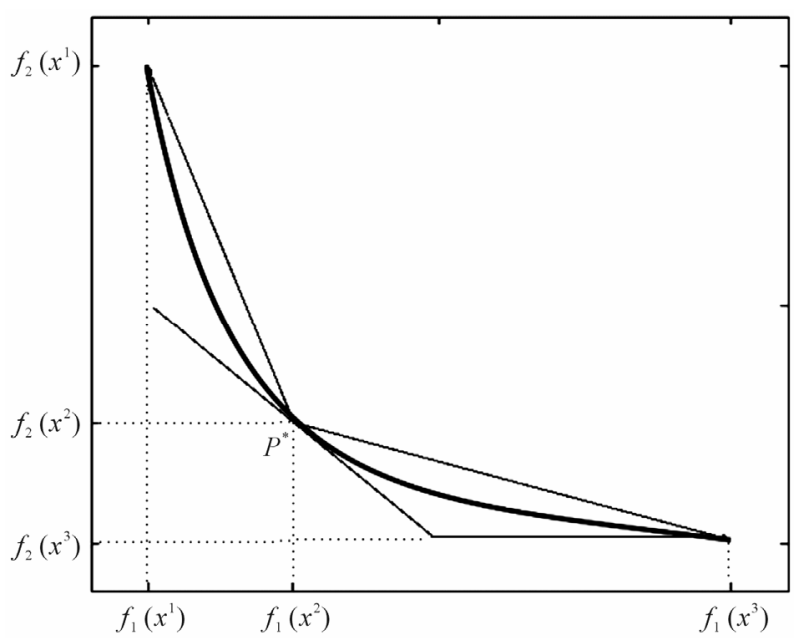

(b)

Figure 3. Lower bounds in STA built due to definitions (9) and (12) presented in (a) and (b), respectively.

and Yang and Goh [8]. First, we formulate two following remarks, which show the relation between the considered error measures.

\section{Remark 1}

Suppose that the lower and upper approximation bounds on the interval $\left[f_{1}\left(x^{k}\right), f_{1}\left(x^{k+1}\right)\right]$ have been build according to the definitions (8) and (9) or (15), then we have

$$
\delta_{k}^{M}=\delta_{k}^{H} \sqrt{1+\left(\frac{f_{2}\left(x^{k}\right)-f_{2}\left(x^{k+1}\right)}{f_{1}\left(x^{k+1}\right)-f_{1}\left(x^{k}\right)}\right)^{2}} .
$$

Moreover,

$$
\delta_{k}^{M} \leq \delta_{k}^{H}\left(1+\frac{f_{2}\left(x^{k}\right)-f_{2}\left(x^{k+1}\right)}{f_{1}\left(x^{k+1}\right)-f_{1}\left(x^{k}\right)}\right) .
$$

See Figure 5. 


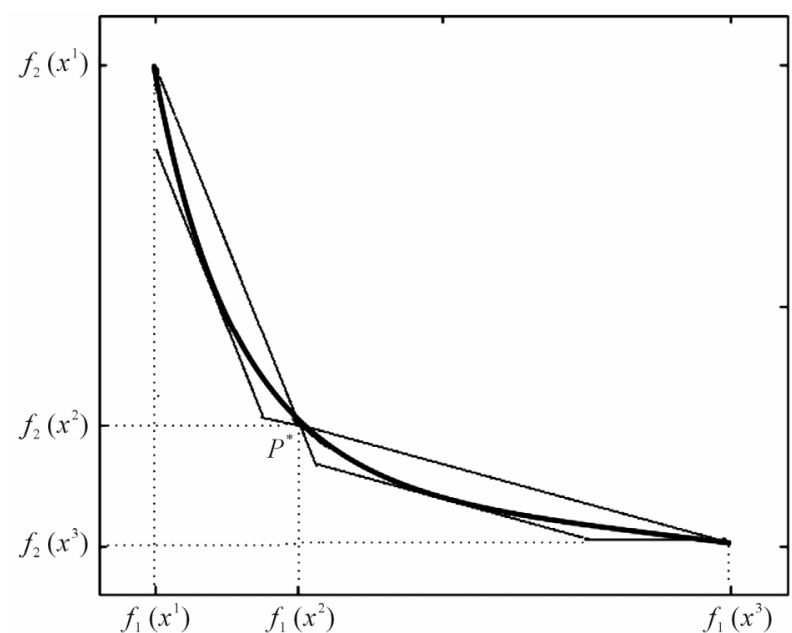

(a)

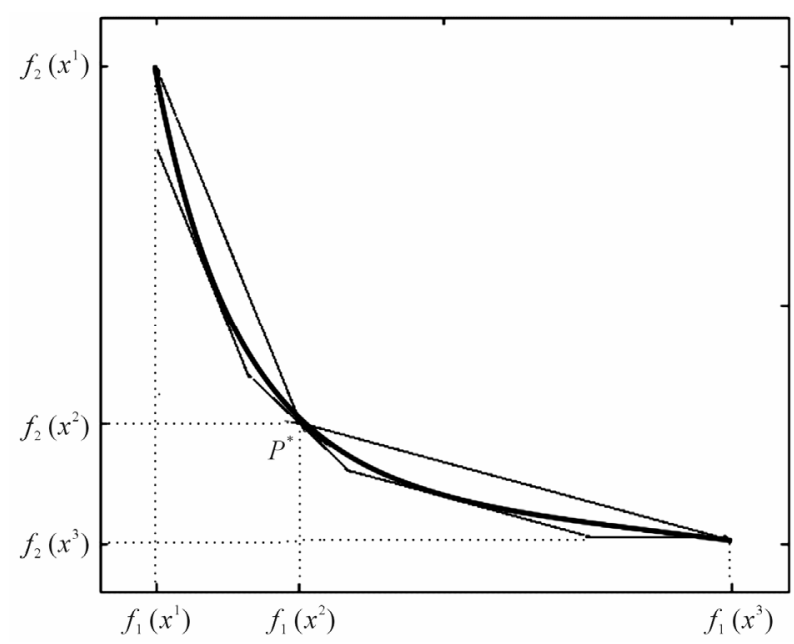

(b)

Figure 4. Lower bounds in TA built due to definitions (15) and (19) presented in (a) and (b), respectively.

\section{Remark 2}

Suppose that the lower and upper approximation bounds on the interval $\left[f_{1}\left(x^{k}\right), f_{1}\left(x^{k+1}\right)\right]$ have been build according to the definitions (8) and (9) or (15), then we have

$$
\delta_{k}^{U} \leq \delta_{k}^{H} \sqrt{\left(f_{1}\left(x^{k}\right)-f_{1}\left(x^{k+1}\right)\right)^{2}+\left(f_{2}\left(x^{k}\right)-f_{2}\left(x^{k+1}\right)\right)^{2}} .
$$

Now, we suppose that the efficient frontier of problem (3) is given as a convex function $g:[a, b] \rightarrow R$ and the one-sided derivatives $g^{+}(a)$ and $g^{-}(b)$ have been evaluated.

The following theorem based on Remark 3 and Theorem 1 in [8], Theorem 2 in [9] and Remark 1 and Remark 2 shows the quadratic convergence property of TA.

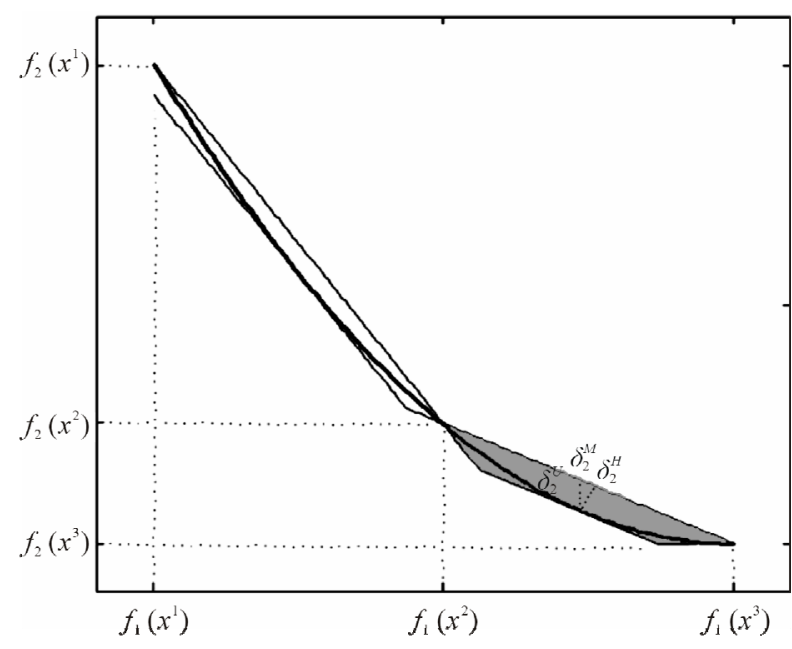

Figure 5. Illustration of error measures defined by (25)-(27).

\section{Theorem 1}

Let $L=b-a$ and $\Delta=g^{-}(b)-g^{+}(a)$ and suppose that the point $(c, g(c))$ was chosen to satisfy the following inequality

$$
c \leq a+\epsilon \frac{b-a}{g(a)-g(b)} .
$$

The number $H$ of optimization problems (16) which have to be solved in order to obtain the Hausdorff distance between upper and lower bounds in TA smaller than or equal to $\epsilon$ satisfies the following inequality

$$
H \leq \max \left\{2\left[\sqrt{\frac{L \Delta}{\epsilon}}\right\rceil-1,3\right\} .
$$

The third point $(c, g(c))$ on the efficient frontier has to be chosen if we want to avoid the problems with the leftmost interval in which the Hausdorff distance between the approximation bounds is equal to the maximum error measure between them. The explanation how to determine a point with property (34) is given in the proof of Theorem 2.

\section{Corollary 1}

Let $L=b-a, \Delta=g^{-}(b)-g^{+}(a)$. The number $M$ of optimization problems (16) which have to be solved in order to obtain the Maximum error between upper and lower bounds in TA smaller than or equal to $\epsilon$ satisfies the following inequality

$$
M \leq \max \left\{2\left[\sqrt{\frac{L \Delta}{\epsilon}(1+\gamma)}\right\rceil-1,3\right\},
$$

where $\gamma=\frac{g(b)-g(a)}{a-b}$.

The number $U$ of optimization problems (16) which have to be solved in order to obtain the Uncertainty area error between upper and lower bounds in TA smaller than or equal to $\epsilon$ satisfies the following inequality 


$$
U \leq \max \left\{2\left\lceil\sqrt{\frac{L \Delta \eta}{\epsilon}}\right\rceil-1,3\right\},
$$

where $\eta=\sqrt{(g(a)-g(b))^{2}+(a-b)^{2}}$.

Yang and Goh [8] noticed that the right directional derivative $g^{+}(a)$ may be close to $-\infty$, that is why using the fact that the Hausdorff distance is invariant under rotation it is better to consider the efficient frontier rotated by $\frac{\pi}{4}$ with the modified directional derivatives $\bar{g}^{+}(\bar{a})$ and $\bar{g}^{-}(\bar{b})$ and with $\bar{L}=\bar{b}-\bar{a}$ as the projective distance of the segment between points $(a, g(a))$ and $(b, g(b))$ onto the line $m(x)=-x$.

Also for STA we may find the upper bound for the number of optimization problems (16) or (28) which have to be solved. First, let us formulate the following lemma.

\section{Lemma 3}

Suppose that the convex function $g:[a, b] \rightarrow R$ is approximated from below by two linear functions $l_{a c}(\cdot)$ and $l_{c b}(\cdot)$ such that $l_{a c}(a)=g(a), l_{c b}(b)=g(b)$ and $l_{a c}(c)=l_{c b}(c)$ for some $c \in(a, b)$. Let $m_{a c}(\cdot)$ and $m_{c b}(\cdot)$ be the lines which intersect the points $(a, g(a))$, $(c, g(c))$ and $(c, g(c)),(b, g(b))$, respectively (see Figure 6). If we denote by $\tan \alpha, \tan \beta, \tan \gamma, \tan \delta$ the slopes of lines $l_{a c}(\cdot), l_{c b}(\cdot), m_{a c}(\cdot), m_{c b}(\cdot)$, respectively, then the following inequality is satisfied

$$
\Delta_{1} L_{1}+\Delta_{2} L_{2} \leq \Delta L
$$

where $\Delta_{1}=\tan \delta-\tan \alpha, \Delta_{2}=\tan \beta-\tan \gamma$, $\Delta=\tan \beta-\tan \alpha, L_{1}=c-a, L_{2}=b-c$ and $L=b-a$.

Proof: See Appendix.

Note that $\Delta_{1}$ and $\Delta_{2}$ are the differences of the slopes of lower approximation functions computed according to
STA for interval $[a, c]$ and $[c, b]$, respectively, and $L_{1}$ and $L_{2}$ are the lengths of these intervals.

The next theorem based on Lemma 5, Theorem 2 from [9] and Lemma 3 establishes the linear convergence property of STA.

\section{Theorem 2}

Let $(a, g(a)),(c, g(c))$ and $(b, g(b))$ are three initial points which are necessary to start STA and suppose that $(c, g(c))$ was chosen to satisfy the following inequality

$$
c \leq a+\epsilon \frac{b-a}{g(a)-g(b)} .
$$

Let $L=b-a$ and let $\Delta=-g^{+}(a)$, then the number $H$ of convex optimization problems ((16) or (28)), which have to be solved in order to make the Hausdorff distance between upper and lower bound in STA with the chord rule or the maximum error rule smaller than or equal to $\epsilon$ satisfies the following inequality

$$
H \leq \max \left\{\left\lceil\frac{L \Delta}{\epsilon}\right\rceil-4,0\right\} .
$$

Proof: See Appendix.

\section{Corollary 2}

Let $(a, g(a)),(c, g(c))$ and $(b, g(b))$ are three initial points that are necessary to start STA and suppose that $(c, g(c))$ was chosen to satisfy the inequality (39). Let $L=b-a$ and let $\Delta=-g^{+}(a)$. The number $M$ of optimization problems (16) which have to be solved in order to obtain the Maximum error between upper and lower bounds in TA smaller than or equal to $\epsilon$ satisfies the following inequality

$$
M \leq \max \left\{\left\lceil\frac{L \Delta}{\epsilon}(1+\gamma)\right\rceil-4,0\right\},
$$

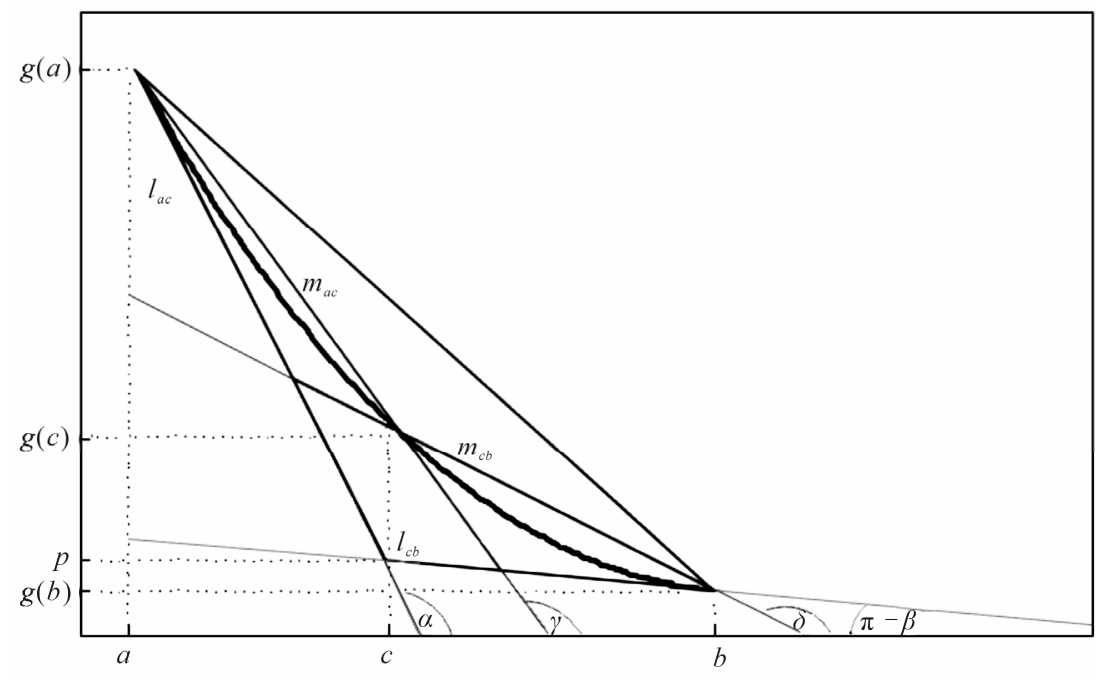

Figure 6. Illustration of functions and angels considered in Lemma 3. 
where $\gamma=\frac{g(b)-g(a)}{a-b}$. The number $U$ of optimization problems (16) which have to be solved in order to obtain the Uncertainty area error between upper and lower bounds in TA smaller than or equal to $\epsilon$ satisfies the following inequality

$$
U \leq \max \left\{\left\lceil\frac{L \Delta \eta}{\epsilon}\right\rceil-4,0\right\},
$$

where $\eta=\sqrt{(g(a)-g(b))^{2}+(a-b)^{2}}$.

\section{Remark 3}

Note that this theorem is true for every convex function $g:[a, b] \rightarrow R$ such that the one-sided derivative $g^{+}(a)$ has been evaluated. If we do not have the derivative information using TA with maximum error rule gives us the linear convergence property of this procedure.

The following theorem by Rote [9] establishes the quadratic convergence property of STA with the modified lower bound as in (12).

\section{Theorem 3}

Let $(a, g(a)),(c, g(c))$ and $(b, g(b))$ are three initial points which are necessary to start STA and suppose that $(c, g(c))$ was chosen to satisfy the following inequality

$$
c \leq a+\epsilon \frac{b-a}{g(a)-g(b)} .
$$

Let $L=b-a$ and let $\Delta=-g^{+}(a)$, then the number $H$ of convex optimization problems (16), which have to be solved in order to make the Hausdorff distance between upper and lower bound in STA and TA with the modified lower bounds and the chord rule smaller than or equal to $\epsilon$ satisfies the following inequality

$$
H \leq \max \left\{\left[\sqrt{\frac{L \Delta}{\epsilon}}\right]-2,0\right\} .
$$

\section{Examples}

In this section we define the stochastic minimum cost flow problem with the moment bicriterion and present two numerical examples, which illustrate algorithm presented in Section 3. Similar to the classic bicriteria network cost flow problem, we consider the directed network $G$ with $n$ nodes and $m$ arcs with the node-arc incidence matrix $D \in R^{n \times m}$, the net outflow vector $b \in R^{n}$ and the capacity bounds $c_{l}, c_{u} \in R_{+}^{m}$. Suppose that the cost per unit of flow through the arc $i \in\{1, \cdots, m\}$ is described by the random variable $C_{i}: \Omega \rightarrow R_{+}$and assume that each variable $C_{i}$ has positive expected value $E\left[C_{i}\right]=c_{i}$ and let $C$ be a random vector such that $\boldsymbol{C}=\left[C_{1}, \cdots, C_{m}\right]^{T}$. The stochastic minimum cost flow problem with the moment bicriterion (SMCFP) is defined as follows

$$
\begin{aligned}
& \min \left[E\left[\boldsymbol{C}^{T} x\right], E[f(\boldsymbol{C}, x)]\right]^{T} \\
& \text { s.t. } D x=b, \\
& c_{l} \leq x \leq c_{u},
\end{aligned}
$$

where $f: R_{+}^{m} \times R_{+}^{m} \rightarrow R_{+}$is the continuous function such that $f(a, \cdot): R_{+}^{m} \rightarrow R_{+}$is convex for every $a \in R_{+}^{m}$.

Since $x \rightarrow E\left[C^{T} x\right]$ and $x \rightarrow E[f(C, x)]$ are linear and convex functions, respectively, then using Lemma 1 we find that the efficient frontier of problem (45) is a convex curve in $R^{2}$.

The following two examples include the comparison of the results obtained by STA, TA with Yang and Goh's method [8] and the Siem et al.'s algorithm [7].

\section{Example 1}

We consider the simple stochastic minimum cost flow problem

$$
\min \left[E\left[\boldsymbol{C}^{T} x\right], f(x)\right]^{T}
$$

s.t.

$$
\begin{array}{lll}
2 \leq x_{1} \leq 6, & 2 \leq x_{2} \leq 6, & 0 \leq x_{3} \leq 4, \\
2 \leq x_{4} \leq 6, & 2 \leq x_{5} \leq 6, & x_{1}+x_{2}=8, \\
x_{1}=x_{3}+x_{4}, & x_{2}+x_{3}=x_{5}, & x_{4}+x_{5}=8,
\end{array}
$$

where $E\left[C_{1}\right]=3, \quad E\left[C_{2}\right]=6, \quad E\left[C_{3}\right]=1, \quad E\left[C_{4}\right]=4$, $E\left[C_{5}\right]=2$ and

$$
f(x)=e^{\frac{x_{1}}{2}}+e^{\frac{x_{2}}{6}}+e^{x_{3}}+e^{\frac{x_{4}}{2}}+e^{\frac{x_{5}}{5}} .
$$

The vectors $x^{1}=(6,2,4,2,6)$ and $x^{3}=(2,6,0,2,6)$ are the lexicographical minima due to the first and the second criterion of problem (46) and

$$
P_{1}=\left(f_{1}\left(x^{1}\right), f_{2}\left(x^{1}\right)\right)=(54,82.12)
$$

and

$$
P_{3}=\left(f_{1}\left(x^{3}\right), f_{2}\left(x^{3}\right)\right)=(62,12.47)
$$

are corresponding to these vectors points on the efficient frontier. To avoid the problem with the leftmost interval we have taken $P_{2}=(54.15,74.63)$ to be the third point of the efficient frontier necessary to start STA and TA.

The results' comparison of subsequent calls of TA and Yang and Goh's algorithm (YG) and the values of the Uncertainty area measure $\delta^{U}$ after each step of TA with the lower bounds defined in (19) (TA1) are presented in Table 1. The results of STA, when new points are computed according to the maximum error rule in common with the Maximum error measure (STAM) and when new points are computed according to the chord rule in common with the Hausdorff measure with the lower bounds defined by the Equation (9) (STAH) and 
Table 1. The results of subsequent calls of TA and Yang and Goh's method (YG) for the problem described in example 1.

\begin{tabular}{cccccccc}
\hline & & \multicolumn{3}{c}{$\mathrm{TA}$} & \multicolumn{3}{c}{$\mathrm{YG}$} \\
\cline { 3 - 8 } Step & $\delta^{H}$ & $\delta^{M}$ & $\delta^{U}$ & $\delta^{U}$ & $\delta^{H}$ & $\delta^{M}$ & $\delta^{U}$ \\
\hline 1 & $\mathbf{2 . 9 9 5}$ & 23.905 & 93.828 & 93.828 & 23.905 & 23.905 & 180.927 \\
2 & $\mathbf{1 . 3 7 6}$ & 9.638 & 25.659 & 23.644 & 5.308 & 9.638 & 31.003 \\
3 & $\mathbf{0 . 5 0 5}$ & 9.638 & 23.591 & 18.781 & 1.497 & 9.638 & 16.269 \\
4 & $\mathbf{0 . 4 9 0}$ & 2.069 & 3.211 & 2.975 & 1.876 & 2.069 & 3.731 \\
5 & $\mathbf{0 . 2 5 1}$ & 2.069 & 3.134 & 2.641 & 1.876 & 2.069 & 3.428 \\
6 & $\mathbf{0 . 1 5 2}$ & 2.069 & 2.805 & 2.641 & 1.876 & 2.069 & 2.398 \\
7 & $\mathbf{0 . 1 3 3}$ & 2.069 & 2.746 & 2.114 & 0.397 & 2.069 & 1.295 \\
8 & $\mathbf{0 . 0 9 6}$ & 2.069 & 2.746 & 2.114 & 0.397 & 2.069 & 1.295 \\
9 & $\mathbf{0 . 0 7 0}$ & 2.069 & 0.402 & 0.371 & 0.397 & 2.069 & 1.295 \\
10 & $\mathbf{0 . 0 6 4}$ & 2.069 & 0.402 & 0.371 & 0.397 & 2.069 & 1.295 \\
\hline
\end{tabular}

Table 2. The results of subsequent calls of STA with the chord rule (STAH, STAH1) and the maximum error rule (STAM) and Siem el al.'s method with interval bisection rule (SB) for the problem described in example 1.

\begin{tabular}{|c|c|c|c|c|c|c|}
\hline \multirow{2}{*}{ Step } & \multicolumn{3}{|c|}{ STAH } & \multicolumn{3}{|c|}{ STAH1 } \\
\hline & $\delta^{H}$ & $\delta^{M}$ & $\delta^{U}$ & $\delta^{H}$ & $\delta^{M}$ & $\delta^{U}$ \\
\hline 1 & 6.553 & 52.303 & 205.288 & 6.553 & 52.303 & 205.288 \\
\hline 2 & 4.163 & 24.116 & 40.901 & 2.902 & 10.540 & 28.462 \\
\hline 3 & 1.744 & 21.671 & 26.547 & 1.170 & 4.556 & 4.259 \\
\hline 4 & 1.225 & 21.671 & 26.547 & 0.401 & 4.556 & 4.259 \\
\hline 5 & 0.760 & 8.633 & 5.064 & 0.367 & 4.556 & 4.259 \\
\hline 6 & 0.648 & 8.633 & 4.084 & 0.167 & 4.556 & 3.610 \\
\hline 7 & 0.485 & 8.633 & 4.084 & 0.126 & 4.556 & 2.727 \\
\hline 8 & 0.305 & 8.107 & 3.567 & 0.122 & 2.543 & 0.908 \\
\hline 9 & 0.269 & 8.107 & 3.567 & 0.115 & 2.543 & 0.908 \\
\hline 10 & 0.236 & 2.836 & 0.676 & 0.107 & 2.543 & 0.908 \\
\hline \multirow{2}{*}{ Step } & \multicolumn{3}{|c|}{ STAM } & \multicolumn{3}{|c|}{ SB } \\
\hline & $\delta^{H}$ & $\delta^{M}$ & $\delta^{U}$ & $\delta^{H}$ & $\delta^{M}$ & $\delta^{U}$ \\
\hline 1 & 6.553 & 52.303 & 205.288 & 3.321 & 49.630 & 99.260 \\
\hline 2 & 5.283 & 24.961 & 82.440 & 2.389 & 31.580 & 31.580 \\
\hline 3 & 3.335 & 12.086 & 32.670 & 2.389 & 21.090 & 12.878 \\
\hline 4 & 1.952 & 11.916 & 8.199 & 2.389 & 8.710 & 12.878 \\
\hline 5 & 1.952 & 4.728 & 8.199 & 2.389 & 6.439 & 12.878 \\
\hline 6 & 0.912 & 4.460 & 2.925 & 0.934 & 4.230 & 4.230 \\
\hline 7 & 0.912 & 3.122 & 2.925 & 0.934 & 3.057 & 2.126 \\
\hline 8 & 0.912 & 3.019 & 2.925 & 0.934 & 2.814 & 2.126 \\
\hline 9 & 0.912 & 2.274 & 1.838 & 0.934 & 2.730 & 2.126 \\
\hline 10 & 0.912 & 1.715 & 1.838 & 0.934 & 2.126 & 2.126 \\
\hline
\end{tabular}

(12) (STAH1) are summarized in Table 2. In the case of Yang and Goh's method the errors are measured in the intervals set by the breakpoints of the upper bound. Table 2 includes also the results of the method described in [7], which uses the interval bisection method of the computing new points with the Maximum error measure. After each step of algorithm we present the maximum values of three error measures: the Maximum error, the Hausdorff distance and the Uncertainty area. As we can notice TA and TA1 performs better than other algorithms giving in each step the smallest values of the Hausdorff distance measure and the Uncertainty area measure. Moreover, from Table 2 we can conclude that STA with the chord rule and the Hausdorff distance gives smaller values of the Hausdorff measure in each step than to two other algorithms, which give comparable results.

\section{Example 2}

We consider the stochastic minimum cost flow problem in the network with 12 nodes and 17 arcs. We would like to minimize the mean value of the total cost of the flow through the network and the second moment of total cost, that is we solve the following problem

$$
\begin{aligned}
& \min \left[E\left[C^{T} x\right], E\left[\left(\boldsymbol{C}^{T} x\right)^{2}\right]\right]^{T} \\
& \text { s.t. } D x=b, \\
& c_{l} \leq x \leq c_{u},
\end{aligned}
$$

where $D \in R^{12 \times 17}, \quad b^{T}=[10,0, \cdots, 0,-10], c_{l}^{T}=[0, \cdots, 0]$ and $c_{u}^{T}=[6,6, \cdots, 6,6]$ and $C=\left[C_{1}, \cdots, C_{17}\right]^{T}: \Omega \rightarrow R_{+}^{17}$ is the random cost vector such that $C_{i}$ and $C_{j}$ are mutually independent for all $i, j \in A$ such that $i \neq j$. We have chosen the values of $E\left[C_{1}\right], \cdots, E\left[C_{17}\right]$ from the interval $[0,2]$ and the values of the second moments $E\left[C_{1}^{2}\right], \cdots, E\left[C_{17}^{2}\right]$ from the interval $[1,3]$. The points on the efficient frontier corresponding to the lexicographical minima of problem (48) and the second criterion are $P_{1}=(62.244,6072.87)$ and $P_{2}=(65.293,5145.63)$. In this example only TA and the Yang and Goh's method are considered, since from Example 1. follows that these two algorithms work faster in comparison to STA and Siem et al.'s method. Table 3 includes the comparison of TA with the method presented in [8]. After each step of the considered methods we present the values of Hausdorff distance, Maximum error and Uncertainty area measure and a new evaluated point. As we can notice TA performs better in compareson to Yang and Goh's algorithm giving in each step the smallest value of the Hausdorff distance between upper and lower approximation bounds.

\section{Conclusions}

Two sandwich algorithms (the Simple Triangle Algorithm 
Table 3. The results of subsequent calls of TA and Yang and Goh's method (YG) for the problem described in example 2.

\begin{tabular}{|c|c|c|c|c|c|c|c|}
\hline \multirow{2}{*}{ Step } & \multirow{2}{*}{$\left(f_{1}\left(x^{*}\right), f_{2}\left(x^{*}\right)\right)$} & \multicolumn{3}{|c|}{ TA } & \multicolumn{3}{|c|}{ YG } \\
\hline & & $\delta^{H}$ & $\delta^{M}$ & $\delta^{U}$ & $\delta^{H}$ & $\delta^{M}$ & $\delta^{U}$ \\
\hline 1 & & 1.507 & 458.259 & 1052.1 & 1.507 & 458.259 & 1397.42 \\
\hline 2 & $(63.410,5259.94)$ & 0.598 & 117.196 & 75.559 & 36.287 & 117.196 & 129.246 \\
\hline 4 & $(64.733,5153.34)$ & 0.168 & 117.196 & 68.847 & 14.101 & 117.196 & 126.457 \\
\hline 5 & $(62.824,5551.23)$ & 0.141 & 29.355 & 8.614 & 28.735 & 29.355 & 17.012 \\
\hline 6 & $(65.013,5147.54)$ & 0.134 & 29.355 & 8.541 & 28.735 & 29.355 & 17.012 \\
\hline 7 & $(63.584,5227.26)$ & 0.071 & 29.355 & 8.541 & 28.735 & 29.355 & 17.012 \\
\hline 8 & $(65.1528,5146.1)$ & 0.058 & 29.355 & 8.534 & 28.735 & 29.355 & 17.012 \\
\hline 9 & $(63.112,5379.36)$ & 0.053 & 29.355 & 8.523 & 7.254 & 29.355 & 15.961 \\
\hline 10 & $(64.438,5163.53)$ & 0.042 & 29.355 & 8.523 & 7.254 & 29.355 & 15.961 \\
\hline
\end{tabular}

and the Trapezium Algorithm) for the approximation of the efficient frontier of the generalized bicriteria minimum cost flow problem have been described. Both methods have been applied in the field of the stochastic minimum cost flow problem with the moment bicriterion.

The Simple Triangle Algorithm uses the lower bound proposed by Siem et al. in [7] with the maximum error rule or the chord rule, what causes faster decrease of the Maximum error measure and the Hausdorff distance measure and, as a result, reduces the number of steps of algorithm in comparison to the Siem et al.'s method. We have established the linear convergence property of this algorithm with both partition rules. If the lower bound in the Simple Triangle Algorithm is defined as in [9], according to the definition (12) and new points of the efficient frontier are computed due to the chord rule then we have the quadratic convergence property of the algorithm.

From the numerical examples follows that the Trapezium Algorithm performs better in comparison to all of the mentioned derivative free algorithms (Siem et al.'s method, Yang and Goh's method) and gives in each step the smallest values of the Hausdorff distance measure between lower and upper bound. It also works faster than Rote's triangle algorithm. Moreover, Trapezium Algorithm may be used for approximation of any convex function without the assumption that the change of the direction of the tangents of this function is less than or equal to $\frac{\pi}{2}$, see [8]. The quadratic convergence property of the Trapezium Algorithm has been established.

For further research we are interested in the construc- tion of a method for solving the stochastic minimum cost flow problem with the moment multicriterion.

\section{REFERENCES}

[1] A. Sedeno-Noda and C. Gonzalez-Martin, "The Biobjective Minimum Cost Flow Problem," European Journal of Operational Research, Vol. 124, No. 3, 2000, pp. 591600. doi:10.1016/S0377-2217(99)00191-5

[2] X. Q. Yang and C. J. Goh, “Analytic Efficient Solution Set for Multi-Criteria Quadratic Programs," European Journal of Operational Research, Vol. 92, No. 1, 1996, pp. 166-181. doi:10.1016/0377-2217(95)00040-2

[3] G. Ruhe, "Complexity Results for Multicriterial and Parametric Network Flows Using a Pathological Graph of Zadeh," Zeitschrift für Operations Research, Vol. 32, No. 1, 1988, pp. 9-27. doi:10.1007/BF01920568

[4] N. Zadeh, "A Bad Network for the Simplex Method and Other Minimum Cost Flow Algorithms," Mathematical Programming, Vol. 5, No. 1, 1973, pp. 255-266. doi:10.1007/BF01580132

[5] R. E. Burkard, H. W. Hamacher and G. Rote, "Sandwich Approximation of Univariate Convex Functions with an Applications to Separable Convex Programming," Naval Research Logistics, Vol. 38, 1991, pp. 911-924.

[6] B. Fruhwirth, R. E. Burkard and G. Rote, “Approximation of Convex Curves with Application to the Bi-Criteria Minimum Cost Flow Problem," European Journal of Operational Research, Vol. 42, No. 3, 1989, pp. 326-338. doi:10.1016/0377-2217(89)90443-8

[7] A. Y. D. Siem, D. den Hertog and A. L. Hoffmann, "A Method for Approximating Univariate Convex Functions Using Only Function Value Evaluations," Center Discussion Paper, Vol. 67, 2007, pp. 1-26.

[8] X. Q. Yang and C. J. Goh, "A Method for Convex Curve 
Approximation," European Journal of Operational Research, Vol. 97, No. 1, 1997, pp. 205-212.

doi:10.1016/0377-2217(95)00368-1

[9] G. Rote, "The Convergence Rate of the Sandwich Algo- rithm for Approximating Convex Functions," Computing, Vol. 48, No. 3-4, 1992, pp. 337-361.

doi: $10.1007 / \mathrm{BF} 02238642$ 


\section{Appendix}

We present the proofs of Lemma 1, Lemma 2, Lemma 3 and Theorem 2.

\section{Proof of Lemma 1}

Let $f_{1}(\cdot)=h^{-1} \circ h_{1}(\cdot), f_{2}(\cdot)=h^{-1} \circ h_{2}(\cdot)$ and let $P_{k}=\left(f_{1}\left(x^{k}\right), f_{2}\left(x^{k}\right)\right)$ for $k=1,2$ be two given points on the efficient frontier of problem (3) and let $\lambda \in[0,1]$.

If $f_{1}\left(x^{1}\right)=f_{1}\left(x^{2}\right)$ then for all $b$ such that $f_{2}\left(x^{1}\right) \geq b \geq f_{2}\left(x^{2}\right)$ the point $\left(f_{1}\left(x^{k}\right), b\right)$ lies also on the efficient frontier of problem (3).

Suppose now that $c^{T} x^{1}=f_{1}\left(x^{1}\right) \neq f_{1}\left(x^{2}\right)=c^{T} x^{2}$. Note that for

$$
\begin{gathered}
C=\left\{x \in X: c^{T} x=\lambda c^{T} x^{1}+(1-\lambda) c^{T} x^{2}\right\}, \\
B_{1}=\left\{y \in X: c^{T} y=c^{T} x^{1}\right\}
\end{gathered}
$$

and

$$
B_{2}=\left\{z \in X: c^{T} z=c^{T} x^{2}\right\},
$$

we have $\lambda B_{1}+(1-\lambda) B_{2} \subseteq C$, what yields

$$
\min _{x \in C} f_{2}(x) \leq \min _{y \in B_{1}, z \in B_{2}} f_{2}(\lambda y+(1-\lambda) z) \text {. }
$$

Due to the convexity of the function $f_{2}$ we have

$$
\begin{aligned}
\min _{x \in C} f_{2}(x) & \leq \min _{y \in B_{1}, z \in B_{2}} f_{2}(\lambda y+(1-\lambda) z) \\
& \leq \min _{y \in B_{1}} \lambda f_{2}(y)+\min _{z \in B_{2}}(1-\lambda) f_{2}(z),
\end{aligned}
$$

what proves the convexity of the efficient frontier of problem (3).

Proof of Lemma 2

Let $x \in X_{E}$, then we have

$$
l\left(f_{1}(x)\right) \leq f_{2}(x) \leq u\left(f_{1}(x)\right),
$$

what is equivalent to the following inequality

$$
l\left(h^{-1}\left(h_{1}(x)\right)\right) \leq h^{-1}\left(h_{2}(x)\right) \leq u\left(h^{-1}\left(h_{1}(x)\right)\right)
$$

and from the fact that $h$ is increasing we have

$$
\begin{aligned}
\bar{l}\left(h_{1}(x)\right) & \left.=h\left(l\left(h^{-1}\left(h_{1}(x)\right)\right)\right)\right) \leq h_{2}(x) \\
& \leq h\left(u\left(h^{-1}\left(h_{1}(x)\right)\right)\right)=\bar{u}\left(h_{1}(x)\right) .
\end{aligned}
$$

Moreover,

$$
\begin{aligned}
& \bar{u}\left(h_{1}(x)\right)-\bar{l}\left(h_{1}(x)\right) \\
= & h\left(u\left(h^{-1}\left(h_{1}(x)\right)\right)\right)-h\left(l\left(h^{-1}\left(h_{1}(x)\right)\right)\right) \\
= & h\left(u\left(f_{1}(x)\right)\right)-h\left(l\left(f_{1}(x)\right)\right) \\
\leq & \max _{y \in\left[l\left(f_{1}\left(x^{r}\right)\right), u\left(f_{1}\left(x^{1}\right)\right)\right]} h(y)\left[u\left(f_{1}(x)\right)-l\left(f_{1}(x)\right)\right] \\
= & \max _{y \in\left[f_{2}\left(x^{r}\right), f_{2}\left(x^{1}\right)\right]} h(y)\left[u\left(f_{1}(x)\right)-l\left(f_{1}(x)\right)\right] \\
= & h\left(f_{2}\left(x^{1}\right)\right)\left[u\left(f_{1}(x)\right)-l\left(f_{1}(x)\right)\right] \\
= & h_{2}\left(x^{1}\right)\left[u\left(f_{1}(x)\right)-l\left(f_{1}(x)\right)\right] .
\end{aligned}
$$

\section{Proof of Lemma 3}

Let $c=\lambda a+(1-\lambda) b$, where $\lambda \in(0,1)$ and let $l_{a c}(c)=l_{c b}(c)=p$. Without loss of generality we may assume that $g(a) \geq g(b)$, then we have $g(a) \geq g(c)$.

From the equalities

$$
\begin{aligned}
& \tan \alpha=\frac{p-g(a)}{c-a}, \tan \beta=\frac{g(b)-p}{b-c}, \\
& \tan \gamma=\frac{g(c)-g(a)}{c-a}, \tan \delta=\frac{g(b)-g(c)}{b-c}
\end{aligned}
$$

follows

$$
\begin{aligned}
& \Delta=\frac{g(b)-p}{\lambda(b-a)}-\frac{p-g(a)}{(1-\lambda)(b-a)}, L=b-a \\
& \Delta_{1}=\frac{g(b)-g(c)}{\lambda(b-a)}-\frac{p-g(a)}{(1-\lambda)(b-a)}, L_{1}=(1-\lambda)(b-a) \\
& \Delta_{2}=\frac{g(b)-p}{\lambda(b-a)}-\frac{g(a)-g(c)}{(1-\lambda)(b-a)} \text { and } L_{2}=\lambda(b-a)
\end{aligned}
$$
to

Now, it is easy to show that inequality (38) is equivalent

$$
\left(2 \lambda^{2}-2 \lambda+1\right)(g(c)-p) \geq 0 .
$$

Using the fact that $g(c) \geq p$ we complete the proof.

\section{Proof of Theorem 2}

Suppose that we have found point $(c, f(c))$ with property (39). It is possible to find such a point as a solution of a convex programing problem with one additional constraint similar to problem (6). From condition (39) follows that $\delta_{1}^{M} \leq \epsilon$ and $\delta_{1}^{H} \leq \epsilon$.

Now, if we consider the interval $[c, b]$, then we have $c-b \leq L$ and

$$
\frac{f(c)-(b)}{b-c} \leq \Delta .
$$

Similar to [9] we prove the theorem by induction on number $N=N\left(\frac{L \Delta}{\epsilon}\right)$.

The induction basis, $N\left(\frac{L \Delta}{\epsilon}\right)=0$, is equivalent to Lemma 1 from [5], which also holds for lower approximation function built according to definition (9).

Suppose that $N \geq 1$. If after one step of STA $\delta \leq \epsilon$, then we have had only one additional evaluation and the thesis is true.

In the other case, let $d \in[c, b]$ be the new computed point and let $L_{1}=d-c$ and $L_{2}=b-d$. Let $\Delta_{1}$ and $\Delta_{2}$ denote the slope differences of the linear functions 
building the lower bounds in the interval $[c, d]$ and $[d, b]$, respectively. We can assume without lost of generality that the error $\delta$ exceeds $\epsilon$ in the right subinterval. Lemma 5 and Lemma 1 from [9] used for the lower approximation bounds built due to definition (9) give the following inequalities

$$
\frac{L_{1} \Delta_{1}}{\epsilon}>1
$$

and

$$
\frac{L_{2} \Delta_{2}}{\epsilon}>4 \text {. }
$$

Lemma 3 and inequality (63) gives

$$
\frac{L_{2} \Delta_{2}}{\epsilon}<\frac{L \Delta}{\epsilon} .
$$

Similarly, Lemma 3 and inequality (64) gives

$$
\frac{L_{1} \Delta_{1}}{\epsilon}<\frac{L \Delta}{\epsilon} .
$$

From (65) and (66) we have

$$
N\left(\frac{L_{1} \Delta_{1}}{\epsilon}\right)<N\left(\frac{L \Delta}{\epsilon}\right) \text { and } N\left(\frac{L_{2} \Delta_{2}}{\epsilon}\right)<N\left(\frac{L \Delta}{\epsilon}\right) \text {. }
$$

Now the induction hypothesis can be applied for $N\left(\frac{L_{1} \Delta_{1}}{\epsilon}\right)$ and $N\left(\frac{L_{2} \Delta_{2}}{\epsilon}\right)$ If $N\left(\frac{L_{1} \Delta_{1}}{\epsilon}\right)=0$, then the theorem's thesis follows directly.

Otherwise, from Lemma 3 we have

$$
\begin{aligned}
& \max _{\Delta_{1} L_{1}+\Delta_{2} L_{2} \leq \Delta L, L_{1}+L_{2}=L, L_{1} \Delta_{1}>\epsilon, L_{2} \Delta_{2}>4 \epsilon}\left(1+N\left(\frac{L_{1} \Delta_{1}}{\epsilon}\right)+N\left(\frac{L_{2} \Delta_{2}}{\epsilon}\right)\right) \\
& =\sin _{\Delta_{1} L_{1}+\Delta_{2} L_{2} \leq \Delta L, L_{1}+L_{2}=L, L_{1} \Delta_{1}>\epsilon, L_{2} \Delta_{2}>4 \epsilon}\left(1+\left\lceil\frac{L_{1} \Delta_{1}}{\epsilon}\right\rceil+\left\lceil\frac{L_{2} \Delta_{2}}{\epsilon}\right\rceil-7\right) \\
& \leq\left\lceil\frac{L \Delta}{\epsilon}\right\rceil-6 \leq\left\lceil\frac{L \Delta}{\epsilon}\right\rceil=N\left(\frac{L \Delta}{\epsilon}\right),
\end{aligned}
$$

what ends the proof. 